\title{
Testing for equality of an increasing number of spectral density functions
}

\author{
Javier Hidalgo*and Pedro Souza
}

25 June 2013

\begin{abstract}
Nowadays it is very frequent that a practitioner faces the problem of modelling large data sets. Relevant examples include spatio-temporal or panel data models with large $N$ and $T$. In these cases deciding a particular dynamic model for each individual/population, which plays a crucial role in prediction and inferences, can be a very onerous and complex task.

The aim of this paper is thus to examine a nonparametric test for the equality of the linear dynamic models as the number of individuals increases without bound. The test has two main features: $(a)$ there is no need to choose any bandwidth parameter and $(b)$ the asymptotic distribution of the test is a normal random variable.
\end{abstract}

\section{INTRODUCTION}

It is arguable that one of the ultimate goals of a practitioner is to predict the future or to obtain good inferences about some parameter of interest. To provide either of

${ }^{*}$ The author gratefully acknowledges the research support by a Catedra of Excellence by the Bank of Santander. 
them, knowledge of the dynamic structure of the data plays a crucial role. Often this is done by choosing an $A R M A$ specification via algorithms such as $A I C$ or $B I C$. However, when the practitioner faces a large dimensional data set, such as panel data models with large $N$ and $T$ or spatio-temporal data sets, the problem to identify a particular model for every element of the "population" can be very onerous and time consuming. In the aforementioned cases, it might be convenient to decide, before to embark in such a cumbersome task, whether the dynamic structure is the same across the different populations. This type of scenarios/models can be regarded as an example of the interesting and growing field of high dimensional data analysis. When the number of spectral density functions to compare is finite, there has been some work, see among others Coates and Diggle (1986), Diggle and Fisher (1991) or Detter and Paparoditis (2009) and references therein. In a spatio-temporal data set we can mention the work by Zhu, Lahiri and Cressie (2002). The above work does not assume any particular model for the dynamic structure of the data. In a parametric context, the test is just a standard problem of comparing the equality of a number of parameters. Finally it is worth mentioning the work in a semiparametric set-up by Härdle and Marron (1990) or Pinkse and Robinson (1995), who compare the equality of shapes up to a linear transformation.

In this paper we are interested on a nonparametric test for equality of the dynamic structure of an increasing number of time series. Two features of the tests are as follows. First, although we will not specify any parametric functional form for the dynamics of the data, our test does not require to choose any bandwidth parameter for its implementation, and secondly, the asymptotic distribution of the test is a gaussian random variable, so that inferences are readily available.

More specifically, let $\left\{x_{t, p}\right\}_{t \in \mathbb{Z}, p \in \mathbb{N}}$ be sequences of linear random variables, to be more specific in Condition $C 1$ below. Denoting the spectral density function of the 
pth sequence $\left\{x_{t, p}\right\}_{t \in \mathbb{Z}}$ by $f_{p}(\lambda)$, we are interested in the null hypothesis

$$
H_{0}: \quad f_{p}(\lambda)=f(\lambda) \text { for all } p \geq 1
$$

a.e. in $[0, \pi]$, being the alternative hypothesis

$$
H_{a}: 0<\iota(\mathbf{P})=|\mathcal{P}| / \mathbf{P}<1,
$$

where $\mathcal{P}=\left\{p: \mu\left(\Lambda_{p}\right)>0\right\}$ with $\Lambda_{p}=\left\{\lambda \in[0, \pi]: f_{p}(\lambda) \neq f(\lambda)\right\}$ and " $|\mathcal{A}|$ " denotes the cardinality of the set $\mathcal{A}$ being $\mu(\cdot)$ the Lebesgue measure. Herewith $\mathbf{P}$ denotes the number of individuals in the sample. Thus $\mathcal{P}$ denotes the set of individuals for which $f_{p}(\lambda)$ is different than the "common" spectral density $f(\lambda)$. In this sense $\iota=: \iota(\mathbf{P})$ represents the proportion of sequences $\left\{x_{t, p}\right\}_{t \in \mathbb{Z}}, p \geq 1$ for which $f_{p}(\lambda) \neq f(\lambda)$. One feature of (2) is that the proportion of sequences $\iota$ can be negligible. More specifically, as we show in the next section, the test has nontrivial power under local alternatives such that $\iota(\mathbf{P})=O\left(\mathbf{P}^{1 / 2}\right)$. The situation when $\iota=\iota(\mathbf{P})$ such that $\iota \searrow 0$ can be of interest for, say, classification purposes or when we want to decide if a new set of sequences share the same dynamic structure. Also, it could be interesting to relax the condition that $\mu\left(\Lambda_{p}\right)>0$. This scenario is relevant if we were only concerned about the behaviour of the spectral density function in a neighbourhood of a frequency, say zero. An example of interest could be to test whether the so-called long range parameter is the same across the different sequences, which generalizes work on testing for unit roots in a panel data model with an increasing number of sequences, see for instance Phillips and Moon (1999). However, for the sake of brevity and space, we will examine this topic somewhere else. Finally it is worth mentioning that we envisage that the results given below can be used in other scenarios which are of interest at a theoretical as well as empirical level. Two of these scenarios are: $i$ ) testing for a break in the covariance structure of a sequence of random variables, where the covariance structure of the data is not even known under the null hypothesis, and $i i$ ) 
as exploratory analysis on whether or not there is separability in a spatial-temporal data set, see Fuentes (2006) and references there in, although contrary to the latter manuscripts we are to allow the number of locations to increase to infinity. However, the relevant technical details for the latter problems are beyond the scope of this paper.

We finish this section relating the results of the paper with the problem of classification with functional data sets, which it is a topic of active research. The reason being that this paper tackles the problem of whether a set of curves, spectral density function, are the same or not. Within the functional data analysis framework, this question translates on whether there is some common structure or if we can split the set into several classes or groups. See classical examples in Ferraty and Vieu (2006), although their approach uses nonparametric techniques which we try to avoid so that the issue of how to choose a bandwidth parameter is avoided and/or the "metric" to decide closeness. With this in mind, we believe that our approach can be used for a classification scheme. For instance, in economics, are the dynamics across different industries the same? This, in the language of functional data analysis, is a problem of supervised classification which is nothing more than a modern name to one of the oldest statistical problems: namely to decide if an individual belongs to a particular population. The term supervised refers to the case where we have a "training" sample which has been classified without error. Moreover, we can envisage that our methodology can be extended to problems dealing with functional data in a framework similar to those examined by Chang and Ogden (2009). The latter is being under current investigation elsewhere, when one it is interested on testing in a partial linear model whether we have common trends across individuals/countries, see Zhang et al. (2012) or Degras, et al. (2012) among others for some related examples.

The remainder of the paper is as follows. Next section describes and examines a test for $H_{0}$ in $(1)$ and we discuss the regularity conditions. Also we discuss the type 
of local alternatives for which the test has no trivial power. The proof of our main results are confined to Sections 3 and 4 . The paper finishes with a conclusion section.

\section{THE TEST AND REGULARITY CONDITIONS}

We begin describing our test. To that end, denote the periodogram of $\left\{x_{t, p}\right\}_{t=1}^{n}$, $p=1, \ldots, \mathbf{P}$, by

$$
I_{p}(j)=\frac{1}{n}\left|\sum_{t=1}^{n} x_{t, p} e^{-i t \lambda_{j}}\right|^{2},
$$

where $\lambda_{j}=2 \pi j / n, j=1, \ldots,[n / 2]=: \tilde{n}$, are the Fourier frequencies.

Suppose that we were interested in $H_{0}$ but only at a particular frequency, say $\lambda_{j}$, for some integer $j=1, \ldots, \tilde{n}$. Then, we might employ

$$
\mathcal{T}_{n}(j)=\frac{1}{\mathbf{P}} \sum_{p=1}^{\mathbf{P}}\left(\frac{I_{p}(j)}{\mathbf{P}^{-1} \sum_{q=1}^{\mathbf{P}} I_{q}(j)}-1\right)^{2}
$$

to decide whether or not $f_{p}(j)=f(j)$. The motivation is that as $\mathbf{P} \nearrow \infty$,

$$
\frac{1}{\mathbf{P}} \sum_{q=1}^{\mathbf{P}} I_{q}(j) \stackrel{P}{\rightarrow} \lim _{\mathbf{P} \rightarrow \infty} \frac{1}{\mathbf{P}} \sum_{q=1}^{\mathbf{P}} f_{q}(j)=: \bar{f}(j),
$$

under suitable regularity conditions. So, under $H_{0}$, we can expect that the sequence of random variables

$$
\left(\frac{1}{\mathbf{P}} \sum_{q=1}^{\mathbf{P}} I_{q}(j)\right)^{-1} I_{p}(j)-1
$$

will have a "mean" equal to zero. On the other hand, under $H_{a}$, for all $p \in \mathcal{P}$ and $\lambda_{j} \in \Lambda_{p}$, we have that the last displayed expression develops a mean different than zero since $\mathcal{E}\left(I_{p}(j) / \bar{f}(j)\right) \neq 1$.

Now extending the above argument to every $j \geq 1$, we then test for $H_{0}$ using

$$
\mathcal{T}_{n}=\frac{1}{\tilde{n}} \sum_{j=1}^{\tilde{n}}\left\{\mathcal{T}_{n}(j)-\left(1-\frac{2}{\mathbf{P}}\right)\right\} .
$$


It is worth to discuss the technical reason for the inclusion of the term $(1-2 / \mathbf{P})$ into the right side of (4). For that purpose, recall Barlett's decomposition see Brockwell and Davis (2000), which implies that

$$
\frac{I_{p}(j)}{\mathbf{P}^{-1} \sum_{q=1}^{\mathbf{P}} I_{q}(j)}-1 \simeq \frac{I_{\varepsilon, p}(j)}{\mathbf{P}^{-1} \sum_{q=1}^{\mathbf{P}} I_{\varepsilon, q}(j)}-1 .
$$

Now using standard linearization, see for instance (15), the second moment of the right side of the last displayed expression is $1-\frac{2}{\mathbf{P}}+o\left((n \mathbf{P})^{-1 / 2}\right)$, see the proof of Theorem 1 for some details. In fact, the reason to correct for the term $2 / \mathbf{P}$ is due to the fact that the mean of $I_{\varepsilon, p}(j)$, which is 1 , is estimated via $\mathbf{P}^{-1} \sum_{q=1}^{\mathbf{P}} I_{\varepsilon, q}(j)$. See for instance Phillips and Moon (1999) for similar arguments.

We shall now introduce the regularity conditions.

Condition C1 $\left\{x_{t, p}\right\}_{t \in \mathbb{Z},}, p \in \mathbb{N}$, are mutually independent covariance stationary linear processes defined as

$$
x_{t, p}=\sum_{j=0}^{\infty} b_{j, p} \varepsilon_{t-j, p} ; \sum_{j=0}^{\infty} j\left|b_{j, p}\right|<\infty, \text { with } b_{p, 0}=1,
$$

where $\left\{\varepsilon_{t, p}\right\}_{t \in \mathbb{Z}}, p \in \mathbb{N}$, are iid sequences with $E\left(\varepsilon_{t, p}\right)=0, E\left(\varepsilon_{t, p}^{2}\right)=\sigma_{\varepsilon, p}^{2}$, $E\left(\left|\varepsilon_{t, p}\right|^{\ell}\right)=\mu_{\ell, p}<\infty$ for some $\ell>8$. Finally, we denote the fourth cumulant of $\left\{\varepsilon_{t, p} / \sigma_{\varepsilon, p}\right\}_{t \in \mathbb{Z}}$ by $\kappa_{4, p}, p \in \mathbb{N}$.

Condition C2 For all $p \in \mathbb{N}, f_{p}(\lambda)$ are bounded away from zero in $[0, \pi]$.

Condition C3 $n$ and $\mathbf{P}$ satisfy $\frac{\mathbf{P}}{n}+\frac{n}{\mathbf{P}^{2}} \rightarrow 0$.

Condition $C 1$ is standard and very mild. This condition implies that

$$
f_{p}(\lambda)=\frac{\sigma_{\varepsilon, p}^{2}}{2 \pi}\left|B_{p}(\lambda)\right|^{2}
$$

where $B_{p}(z)=\sum_{j=0}^{\infty} b_{j, p} e^{i j z}$. Thus, under $H_{0}$, we have that

$$
B_{p}(z)=: B(z)=\sum_{j=0}^{\infty} b_{j} e^{i j z}, \quad \text { and } \quad \sigma_{\varepsilon, p}^{2}=\sigma_{\varepsilon}^{2}, \quad p \geq 1
$$


$C 1$ together with $C 2$ implies that the spectral density functions $\left\{f_{p}(\lambda)\right\}, p=$ $1,2, \ldots$, are twice continuously differentiable. We could relax the condition to allow for strong dependent data at the expense of some strengthening of Condition $C 3$. However, since the literature is full of scenarios where results for weakly dependent sequences follows for strong dependence, we have decided to keep $C 1$ as it stands for the sake of clarity. Also it is worth emphasizing that we do not assume that the sequences are identically distributed, as we allow the fourth cumulant to vary among the sequences. It is worth signaling out that $C 2$ implies that the sequences $\left\{x_{t, p}\right\}_{t \in \mathbb{Z}}$, $p \in \mathbb{N}$, have also a autoregression representation given by

$$
x_{t, p}=\sum_{j=1}^{\infty} a_{j, p} x_{t-j, p}+\varepsilon_{t, p} ; \sum_{j=0}^{\infty} j\left|a_{j, p}\right|<\infty
$$

and $f_{p}(\lambda)=\frac{\sigma_{\varepsilon, p}^{2}}{2 \pi}\left|A_{p}(\lambda)\right|^{-2}$, where $A_{p}(z)=1-\sum_{j=1}^{\infty} a_{j} e^{i j z}$. Finally a word regarding Condition $C 1$ is worth considering. We have assumed that the sequences $\left\{x_{t, p}\right\}_{t \in \mathbb{Z}}$ and $\left\{x_{t, q}\right\}_{t \in \mathbb{Z}}$, for all $p, q=1,2, .$. are mutually independent. It is true that this assumption in many settings can be difficult to justify and we can expect some "spatial" dependence among the sequences. An inspection of our proofs indicate that the results would follow provided some type of "weak" dependence. That is, if we denote $\gamma_{p, q}(t)$ the dependence between $\left\{\varepsilon_{t, p}\right\}_{t \in \mathbb{Z}}$ and $\left\{\varepsilon_{t, q}\right\}_{t \in \mathbb{Z}}$, then we have that $\mathbf{P}^{-1} \sum_{p, q=1}^{\mathbf{P}}\left|\gamma_{p, q}(t)\right| \leq C<\infty$ uniformly in $t$. The only main noticeable difference when we compare the results that we should have to those in Theorem 1 below is that the variance of the asymptotic distribution in Theorem 1 below would reflect this dependence among the sequences $\left\{\varepsilon_{t, p}\right\}_{p \in \mathbb{N}}$. However for simplicity and to follow more easily the arguments we have decided to keep $C 1$ as it stands.

Denote

$$
\kappa_{4}=\lim _{\mathbf{P} \rightarrow \infty} \frac{1}{\mathbf{P}} \sum_{p=1}^{\mathbf{P}} \kappa_{4, p}
$$


Theorem 1 Under $H_{0}$ and assuming $C 1-C 3$, we have that

$$
\tilde{n}^{1 / 2} \mathbf{P}^{1 / 2} \mathcal{T}_{n} \rightarrow_{d} \mathcal{N}\left(0,4+\kappa_{4}\right) .
$$

Proof. The proof of this theorem is confined to Section 3 below.

The conclusion that we draw from Theorem 1 is that the asymptotic distribution is standard. However, its asymptotic variance depends on the "average" fourth cumulant $\kappa_{4}$. So, to be able to make inferences, we need to provide a consistent estimator of $\kappa_{4}$. One possibility comes from the well known formula in Grenander and Rosenblatt (1957). However, in our context it can be a computational burden prospect, apart from the fact that all we need is not to obtain a consistent estimator of all $\kappa_{4, p}$ but for the average. Another potential problem to estimate $\kappa_{4}$ via Grenander and Rosenblatt (1957) is that it would need a bandwidth or cut-off point to compute the estimator of $\kappa_{4, p}$, for all $p \geq 1$. This is the case as the computation of $\kappa_{4}$, i.e. $\kappa_{4, p}$, depends the covariance structure of $x_{t, p}^{2}$ as well as that of $x_{t, p}$. This creates the problem of how to choose this bandwidth parameter for each individual sequence. Thus, we propose and examine an estimator of $\kappa_{4}$ which is easy to compute and in addition it will not require the choice of any bandwidth parameter in its computation.

To that end, denote the discrete Fourier transform of a generic sequence $\left\{u_{t}\right\}_{t=1}^{n}$ by

$$
w_{u}(j)=\frac{1}{n^{1 / 2}} \sum_{t=1}^{n} u_{t} e^{-i t \lambda_{j}}, \quad j=1, \ldots, \tilde{n} .
$$

Also, $C 1$ and $H_{0}$ suggest that the discrete Fourier transform of $\left\{\varepsilon_{t, p}\right\}_{t=1}^{n}$ is

$$
w_{\varepsilon, p}(j) \approx A\left(-\lambda_{j}\right) w_{x, p}(j),
$$

where $B^{-1}\left(\lambda_{j}\right)=A\left(\lambda_{j}\right)$ and using the inverse transform of $w_{u}(j)$, we conclude that

$$
\varepsilon_{t, p} \approx \frac{1}{n^{1 / 2}} \sum_{j=1}^{n} e^{i t \lambda_{j}} A\left(-\lambda_{j}\right) w_{x, p}(j)
$$


where " $\approx$ " should be read as "approximately". Notice that the last two expressions are valid under the alternative hypothesis if instead of $A\left(-\lambda_{j}\right)$ we write $A_{p}\left(-\lambda_{j}\right)$.

The latter indicates that the problem to obtain the residuals $\left\{\widehat{\varepsilon}_{t, p}\right\}_{t=1}^{n}$ becomes a problem to compute an estimator of $A\left(-\lambda_{j}\right)$. To that end, denote by

$$
\widehat{f}(j)=\frac{1}{\mathbf{P}} \sum_{p=1}^{\mathbf{P}} I_{p}(j)
$$

the estimator of $f(j)$ under $H_{0}$. Then, we compute $\left\{\widehat{\varepsilon}_{t, p}\right\}_{t=1}^{n}, p=1, \ldots, \mathbf{P}$, as

$$
\widehat{\varepsilon}_{t, p}=\frac{1}{n^{1 / 2}} \sum_{j=1}^{n-1} e^{i t \lambda_{j}} \widehat{A}(j) w_{x, p}(j), \quad\left\{\begin{array}{c}
p=1, \ldots, \mathbf{P} \\
t=1, \ldots, n
\end{array}\right.
$$

where

$$
\begin{aligned}
\widehat{A}(j) & =\exp \left\{-\sum_{r=1}^{n} \widehat{c}_{r} e^{-i r \lambda}\right\}, j=1, \ldots, \tilde{n} \\
\widehat{c}_{r} & =\frac{1}{n} \sum_{\ell=1}^{n} \log \widehat{f}\left(\lambda_{\ell}\right) \cos r \lambda_{\ell}, \quad r=1, \ldots, n .
\end{aligned}
$$

Note that $\widehat{\sigma}_{\varepsilon}=2 \pi \exp \left(\widehat{c}_{0}\right)$. The function $\exp \left\{\sum_{r=1}^{n} \widehat{c}_{r} e^{-i r \lambda}\right\}$ is an estimator of $A(\lambda)=\exp \left\{\sum_{r=1}^{\infty} c_{r} e^{-i r \lambda}\right\}$ with

$$
c_{r}=\frac{1}{\pi} \int_{0}^{\pi} \log f(\lambda) \cos (r \lambda) d \lambda
$$

Observe that $e^{c_{0}}|A(\lambda)|^{-2}=f(\lambda)$ and the motivation to estimate $A(\lambda)$ by $\widehat{A}(j)$ comes from the canonical spectral decomposition of $f(\lambda)$, see for instance Brillinger (1981, p.78 - 79) or Hannan (1970). Moreover, denoting

$$
\widehat{a}_{\ell}=\frac{1}{n} \sum_{j=-\tilde{n}+1}^{\tilde{n}-1} \widehat{A}(j) e^{i \ell \lambda_{j}}, \quad \ell=1, \ldots, n
$$

we could also estimate $A(j)$ as $\widehat{A}(j)=1+\widehat{a}_{1} e^{-i \lambda_{j}}+\ldots+\widehat{a}_{n} e^{-i n \lambda_{j}}$. This comes from the fact that $e^{c_{0}}\left|\exp \left\{\sum_{r=1}^{\infty} c_{r} e^{-i r \lambda}\right\}\right|^{2}=f(\lambda)$ and that $a_{\ell}$ is the $\ell$ th Fourier coefficient of 
$\exp \left\{\sum_{r=1}^{\infty} c_{r} e^{-i r \lambda}\right\}$. In fact, one of the implications of the canonical decomposition is that $\exp \left\{\sum_{r=1}^{\infty} c_{r} e^{-i r \lambda}\right\}=1-\sum_{j=1}^{\infty} a_{j} e^{i j \lambda}$. Also, we might consider $\widehat{a}_{\ell}$ and $\widehat{A}(j)=$ $1+\widehat{a}_{1} e^{-i \lambda_{j}}+\ldots+\widehat{a}_{n} e^{-i n \lambda_{j}}$ as estimators of the average $\bar{a}_{\ell}=\mathbf{P}^{-1} \sum_{p=1}^{\mathbf{P}} a_{p, \ell}$ and $\bar{A}(j)=$ $\mathbf{P}^{-1} \sum_{p=1}^{\mathbf{P}} A_{p}(j)$ respectively, as $\widehat{f}(j)$ is an estimator of $\bar{f}(j)$ under the maintain hypothesis. In addition it is worth pointing out that from a computational and theoretically point of view, we may only estimate the first, say $C n^{1 / 2}, c_{r}$ coefficients as $C 1$ implies that $c_{r}=O\left(r^{-2}\right)$.

Then, we compute our estimator of $\kappa_{4}$ as

$$
\widehat{\kappa}_{4}=\frac{1}{\mathbf{P}} \sum_{p=1}^{\mathbf{P}} \frac{1}{n} \sum_{t=1}^{n}\left(\frac{\widehat{\varepsilon}_{t, p}^{4}}{\widehat{\sigma}_{\varepsilon}^{4}}-3\right) .
$$

Nevertheless we have, as a by-product, that $\frac{1}{n} \sum_{t=1}^{n}\left(\frac{\widehat{\varepsilon}_{t, p}^{4}}{\widehat{\sigma}_{\varepsilon}^{4}}-3\right)$ is an estimator of the fourth cumulant $\kappa_{4, p}, p \geq 1$.

Theorem 2 Under $H_{0}$ and assuming $C 1-C 3$, we have that $\widehat{\kappa}_{4} \rightarrow_{P} \kappa_{4}$.

Proof. The proof of this theorem is confined to Section 4 below.

We finish this section describing the local alternatives for which $\mathcal{T}_{n}$ does not have trivial power. In addition as a corollary to Proposition 1 below, we easily conclude that $\mathcal{T}_{n}$ will then provide a consistent test for $H_{0}$. To that end, we consider the local alternatives

$$
H_{l}:\left\{\begin{array}{l}
f_{p}(\lambda)=f(\lambda)\left(1+\frac{1}{n^{1 / 2}} g_{p}(\lambda)\right) \text { for all } p \leq C \mathbf{P}^{1 / 2} \\
f_{p}(\lambda)=f(\lambda) \quad \text { for all } C \mathbf{P}^{1 / 2}<p \leq \mathbf{P}
\end{array}\right.
$$

where $g_{p}(\lambda)$ is different than zero in $\Lambda_{p}$. So, we hope that the test will have nontrivial power for alternatives converging to the null hypothesis at rate of order $O\left(n^{-1 / 2} \mathbf{P}^{-1 / 2}\right)$, which is the rate that one would expect in a parametric setting. Notice that, without loss of generality, we have ordered the sequences in such a way that the first $C \mathbf{P}^{1 / 2}$ sequences are those for which $f_{p}(\lambda) \neq f(\lambda)$. Assuming for notational simplicity $\Lambda_{p}=\Lambda$, we then have the following result. 
Proposition 3 Under $H_{l}$ and assuming $C 1-C 3$, we have that

$$
\tilde{n}^{1 / 2} \mathbf{P}^{1 / 2} \mathcal{T}_{n} \rightarrow_{d} \mathcal{N}\left(c, 4+\kappa_{4}\right),
$$

where $c=2 \int_{\Lambda} g(\lambda) d \lambda$ with $g(\lambda)=\lim _{\mathbf{P} / \infty} \mathbf{P}^{-1 / 2} \sum_{p=1}^{\mathbf{P}^{1 / 2}} g_{p}(\lambda)$.

Proof. First, proceeding as in the proof of Theorem 1, it easily follows that $\mathcal{T}_{n}$ is governed by the behaviour of

$$
\mathcal{T}_{n}^{1}=\frac{1}{\tilde{n}} \sum_{j=1}^{\tilde{n}}\left\{\frac{1}{\mathbf{P}} \sum_{p=1}^{\mathbf{P}}\left(\frac{f_{p}(j) \stackrel{\circ}{I}_{\varepsilon, p}(j)+f_{p}(j)}{\mathbf{P}^{-1} \sum_{q=1}^{\mathbf{P}} f_{q}(j) \stackrel{\circ}{I}_{\varepsilon, q}(j)+1}-1\right)^{2}-\left(1-\frac{2}{\mathbf{P}}\right)\right\},
$$

where $\stackrel{\circ}{\varepsilon, p}_{\varepsilon}(j)=I_{\varepsilon, p}(j)-1$ and where for notational simplicity we have abbreviated $f_{p}(j) / \bar{f}_{\mathbf{P}}(j)$ as $f_{p}(j)$ with $\bar{f}_{\mathbf{P}}(j)=\mathbf{P}^{-1} \sum_{q=1}^{\mathbf{P}} f_{q}(j)$. Also, to abbreviate the arguments and notation we assume that $\sigma_{\varepsilon, p}^{2}=\sigma_{\varepsilon}^{2}$. Now, using (15) and the arguments in the proof of Theorem 1, we have that

$$
\frac{1}{\left(\mathbf{P}^{-1} \sum_{q=1}^{\mathbf{P}} f_{q}(j) \stackrel{\circ}{I}_{\varepsilon, q}\left(j_{j}\right)+1\right)^{2}} \stackrel{\text { asym }}{\simeq} 1-\left(b_{n}^{2}(j)+2 b_{n}(j)\right),
$$

where $b_{n}(j)=\mathbf{P}^{-1} \sum_{q=1}^{\mathbf{P}} f_{q}(j) \stackrel{\circ}{I}, q_{\varepsilon}(j)$ and “ $\stackrel{\text { asym }}{\simeq}$ " denotes that the left- and right hand sides are asymptotically equivalent.

So, the asymptotic behaviour of $\mathcal{T}_{n}$ is that governed by

$$
\begin{aligned}
& \frac{1}{\tilde{n}} \sum_{j=1}^{\tilde{n}}\left\{\frac{1}{\mathbf{P}} \sum_{p=1}^{\mathbf{P}}\left\{\left(f_{p}(j) \stackrel{\circ}{\varepsilon}, p_{\varepsilon}(j)-b_{n}(j)\right)+\left(f_{p}(j)-1\right)\right\}^{2}\right. \\
& \left.\times\left(1-\left(b_{n}^{2}(j)+2 b_{n}(j)\right)\right)\right\}-\left(1-\frac{2}{\mathbf{P}}\right) .
\end{aligned}
$$

Now, except terms of smaller order of magnitude, the expectation of the last displayed expression is

$$
\begin{aligned}
& \frac{1}{\tilde{n}} \sum_{j=1}^{\tilde{n}}\left\{\left(1-\frac{2}{\mathbf{P}}\right) \frac{1}{\mathbf{P}} \sum_{q=1}^{\mathbf{P}} f_{q}^{2}(j)-1+\mathbf{P}^{-1} \sum_{q=1}^{\mathbf{P}}\left(f_{p}(j)-1\right)^{2}\right\} \\
= & \frac{2}{\tilde{n}} \sum_{j=1}^{\tilde{n}} n^{-1 / 2} \mathbf{P}^{-1 / 2} \sum_{q=1}^{\mathbf{P}^{1 / 2}} g_{q}(j)(1+o(1)),
\end{aligned}
$$


because, recalling the definition of $H_{l}$, we have that, for all $j=1, \ldots, \tilde{n}$,

$$
\frac{1}{\mathbf{P}} \sum_{q=1}^{\mathbf{P}} f_{q}^{2}(j)-1=\frac{1}{\tilde{n}^{1 / 2} \mathbf{P}} \sum_{q=1}^{\mathbf{P}^{1 / 2}} g_{q}(j)(1+o(1)) .
$$

So, standard arguments indicate that $\tilde{n}^{1 / 2} \mathbf{P}^{1 / 2}$ times the right side of (9) is

$$
\frac{2^{1 / 2}}{\tilde{n}} \sum_{j=1}^{\tilde{n}}\left\{\frac{1}{\mathbf{P}^{1 / 2}} \sum_{q=1}^{\mathbf{P}^{1 / 2}} g_{q}(j)\right\} \underset{\mathbf{P}, n / \infty}{\rightarrow} c
$$

since $\bar{f}_{\mathbf{P}}(j)=1+n^{-1 / 2} \mathbf{P}^{-1} \sum_{q=1}^{\mathbf{P}^{1 / 2}} g_{q}(j)$ under $H_{l}$. From here the proof of the proposition proceeds as that of Theorem 1 and so it is omitted.

One immediate conclusion that we draw from Proposition 1 is that our test detect local alternatives shrinking to the null hypothesis at a parametric rate, even being our scenario a nonparametric one.

\section{PROOF OF THEOREM 1}

In what follows we denote $\stackrel{\circ}{I_{\varepsilon, p}}(j)=I_{\varepsilon, p}(j)-1$, where

$$
I_{\varepsilon, p}(j)=\frac{1}{n} \sum_{t, s=1}^{n} \frac{\varepsilon_{t, p}}{\sigma_{\varepsilon}} \frac{\varepsilon_{s, p}}{\sigma_{\varepsilon}} e^{i(t-s) \lambda_{j}} \quad \text { and } \quad R_{p}(j)=\frac{I_{p}(j)}{f(j)}-I_{\varepsilon, p}(j) .
$$

Using Brockwell and Davis's (1991) Theorem 10.3.1 and then $C 1$ we have that standard algebra implies that

$$
\begin{gathered}
\mathcal{E}\left(\frac{1}{\mathbf{P}} \sum_{p=1}^{\mathbf{P}} R_{p}^{2}(j)\right)=O\left(\frac{1}{n}\right) ; \quad \mathcal{E}\left(R_{p}(j)\right)=O\left(\frac{1}{n}\right) \\
\mathcal{E}\left(\frac{1}{\mathbf{P}} \sum_{p=1}^{\mathbf{P}} R_{p}(j)\right)^{2}=O\left(n^{-2}+\mathbf{P}^{-1} n^{-1}\right) .
\end{gathered}
$$


In addition and denoting $\mathcal{I}(\cdot)$ as the indicator function, $C 1$ also implies that

$$
\begin{aligned}
\mathcal{E}\left(\frac{1}{\mathbf{P}} \sum_{p=1}^{\mathbf{P}} \stackrel{\circ}{I}_{\varepsilon, p}(j)\right)^{2} & =O\left(\mathbf{P}^{-1}\right) ; \\
\mathcal{E}\left(\frac{1}{\mathbf{P}} \sum_{p=1}^{\mathbf{P}} \stackrel{\circ}{I}_{\varepsilon, p}(j) \frac{1}{\mathbf{P}} \sum_{p=1}^{\mathbf{P}} \stackrel{\circ}{I}_{\varepsilon, p}(k)\right) & =\frac{\mathcal{I}(j=k)}{\mathbf{P}}+\frac{1}{n \mathbf{P}} \frac{1}{\mathbf{P}} \sum_{p=1}^{\mathbf{P}} \kappa_{4, p} .
\end{aligned}
$$

Next, using (11) and (12), we obtain that

$$
\begin{aligned}
\left(\frac{1}{\mathbf{P}} \sum_{p=1}^{\mathbf{P}} \frac{I_{p}(j)}{f(j)}\right)^{2} & =\left(\frac{1}{\mathbf{P}} \sum_{p=1}^{\mathbf{P}}\left(R_{p}(j)+\stackrel{\circ}{I}_{\varepsilon, p}(j)+1\right)\right)^{2} \\
& =\left(\frac{1}{\mathbf{P}} \sum_{p=1}^{\mathbf{P}} \stackrel{\circ}{\varepsilon}_{\varepsilon, p}(j)\right)^{2}+\frac{2}{\mathbf{P}} \sum_{p=1}^{\mathbf{P}} \stackrel{\circ}{I}_{\varepsilon, p}(j)+1+v_{n},
\end{aligned}
$$

where $v_{n}$ is a sequence of random variables such that $\mathcal{E} v_{n}=O_{p}\left(n^{-1}\right)$. Thus, using the linearization

$$
\frac{1}{a_{n}}=\frac{1}{a}-\frac{a_{n}-a}{a^{2}}+\frac{\left(a_{n}-a\right)^{2}}{a^{3}}+O\left(\left|a_{n}-a\right|^{3}\right),
$$

with $a_{n}=\left(\mathbf{P}^{-1} \sum_{p=1}^{\mathbf{P}} I_{p}(j) / f(j)\right)^{2}$ and $a=1$, it follows easily by $C 3$ and then by (11) that

$$
\begin{aligned}
\mathcal{T}_{n}= & \frac{1}{\tilde{n}} \sum_{j=1}^{\tilde{n}}\left\{\frac { 1 } { \mathbf { P } } \sum _ { p = 1 } ^ { \mathbf { P } } \left[\left(R_{p}(j)+\stackrel{\circ}{I}_{\varepsilon, p}(j)-\frac{1}{\mathbf{P}} \sum_{q=1}^{\mathbf{P}}\left(R_{q}(j)+\stackrel{\circ}{I}_{\varepsilon, q}(j)\right)\right)^{2}\right.\right. \\
& \left.\left.\times\left(1+3\left(\frac{1}{\mathbf{P}} \sum_{q=1}^{\mathbf{P}} \stackrel{\circ}{I}_{\varepsilon, q}(j)\right)^{2}-\frac{2}{\mathbf{P}} \sum_{q=1}^{\mathbf{P}} \stackrel{\circ}{I}_{\varepsilon, q}(j)\right)\right]-\left(1-\frac{2}{\mathbf{P}}\right)\right\} \\
& +o_{p}\left((n \mathbf{P})^{-1 / 2}\right) \\
= & \frac{1}{\tilde{n}} \sum_{j=1}^{\tilde{n}}\left\{\frac { 1 } { \mathbf { P } } \sum _ { p = 1 } ^ { \mathbf { P } } \left[\left(\stackrel{\circ}{I}_{\varepsilon, p}^{2}(j)-\left(\frac{1}{\mathbf{P}} \sum_{q=1}^{\mathbf{P}} \stackrel{\circ}{I}_{\varepsilon, q}(j)\right)^{2}\right)\right.\right. \\
& \left.\left.\times\left(1+3\left(\frac{1}{\mathbf{P}} \sum_{q=1}^{\mathbf{P}} \stackrel{\circ}{\varepsilon}_{\varepsilon, q}(j)\right)^{2}-\frac{2}{\mathbf{P}} \sum_{q=1}^{\mathbf{P}} \stackrel{\circ}{I}_{\varepsilon, q}(j)\right)\right]-\left(1-\frac{2}{\mathbf{P}}\right)\right\} \\
& +o_{p}\left((n \mathbf{P})^{-1 / 2}\right) .
\end{aligned}
$$


From here and observing that $\mathcal{E}\left(\sum_{p=1}^{\mathbf{P}}\left\{\dot{I}_{\varepsilon, p}^{2}(j)-1\right\}\right)^{2}=O\left(\mathbf{P}+n^{-1} \sum_{p=1}^{\mathbf{P}} \kappa_{4, p}\right)$, we obtain by (13) and standard arguments, that

$$
\begin{aligned}
\mathcal{T}_{n}= & \frac{1}{\tilde{n}} \sum_{j=1}^{\tilde{n}}\left\{\frac{1}{\mathbf{P}} \sum_{p=1}^{\mathbf{P}} \stackrel{\circ}{I}_{\varepsilon, p}^{2}(j)-2 \stackrel{\circ}{I}_{\varepsilon, p}(j)+2\left(\frac{1}{\mathbf{P}} \sum_{q=1}^{\mathbf{P}} \stackrel{\circ}{I}_{\varepsilon, q}(j)\right)^{2}-\frac{2}{\mathbf{P}^{2}} \sum_{q, p=1}^{\mathbf{P}} \stackrel{\circ}{I}_{\varepsilon, q}^{2}(j) \stackrel{\circ}{I}_{\varepsilon, p}(j)\right\} \\
& -\left(1-\frac{2}{\mathbf{P}}\right)+o_{p}\left(n^{-1 / 2} \mathbf{P}^{-1 / 2}\right) .
\end{aligned}
$$

We can now see the motivation to include the term $(1-2 / \mathbf{P})$ in (4) as standard manipulations indicate that

$$
\mathcal{E}\left(\frac{1}{\mathbf{P}} \sum_{q=1}^{\mathbf{P}} \stackrel{\circ}{I}_{\varepsilon, q}(j)\right)^{2}-\mathcal{E}\left(\frac{1}{\mathbf{P}^{2}} \sum_{q, p=1}^{\mathbf{P}} \stackrel{\circ}{I}_{\varepsilon, q}^{2}(j) \stackrel{\circ}{I}_{\varepsilon, p}(j)\right)=\frac{1}{\mathbf{P}}+o\left(\frac{1}{\mathbf{P} n}\right) .
$$

Next, $\mathcal{E}\left(\sum_{j=1}^{\tilde{n}}\left\{\left(\mathbf{P}^{-1 / 2} \sum_{p=1}^{\mathbf{P}} \stackrel{\circ}{I}_{\varepsilon, p}(j)\right)^{2}-1\right\}\right)^{2}$ is equal to

$$
\begin{aligned}
& \sum_{j, k=1}^{\tilde{n}} \frac{1}{\mathbf{P}^{2}} \sum_{q 1, q_{2}, p_{1}, p_{2}=1}^{\mathbf{P}}\left\{\mathcal{E}\left(\stackrel{\circ}{I}_{\varepsilon, q_{1}}(j) \stackrel{\circ}{I}_{\varepsilon, q_{2}}(j) \stackrel{\circ}{I}_{\varepsilon, p_{1}}(k) \stackrel{\circ}{I}_{\varepsilon, p_{2}}(k)\right)-1\right\} \\
= & \sum_{j, k=1}^{\tilde{n}} \frac{1}{\mathbf{P}^{2}} \sum_{q, p=1}^{\mathbf{P}}\left[\mathcal{E} \stackrel{\circ}{I}_{\varepsilon, q}^{2}(j) \mathcal{E} \stackrel{\circ}{I}_{\varepsilon, p}^{2}(k)-1\right]+2\left(\frac{1}{\mathbf{P}} \sum_{p=1}^{\mathbf{P}} \mathcal{E}\left\{\stackrel{\circ}{I}_{\varepsilon, p}(j) \stackrel{\circ}{I}_{\varepsilon, p}(k)\right\}\right)^{2} \\
& +\frac{1}{\mathbf{P}^{2}} \sum_{p=1} \mathcal{E}\left\{\stackrel{\circ}{I}_{\varepsilon, p}^{2}(j) \stackrel{\circ}{I}_{\varepsilon, p}^{2}(k)\right\}+O\left(\frac{n^{2}}{\mathbf{P}}\right)
\end{aligned}
$$

because $C 1$ implies that the expectation on the left of the last displayed equality is zero unless the subindexes $\left(p_{1}, p_{2}, q_{1}, q_{2}\right)$ comes by pairs. Now using Brillinger's (1980) Theorems 2.3.2 and 4.3.1., and in particular expressions in (2.3.7) and (4.3.15)), we obtain that $\operatorname{Cov}\left(I_{\varepsilon, p}(j), I_{\varepsilon, p}(k)\right)=\left(1+O\left(n^{-1}\right)\right) \mathcal{I}(j=k)+O\left(n^{-1}\right) \mathcal{I}(j \neq k)$. The latter implies that the right side of the last displayed equation is $O\left(n^{2} / \mathbf{P}\right)$. Similarly

$$
\mathcal{E}\left(\sum_{j=1}^{\tilde{n}}\left\{\frac{1}{\mathbf{P}} \sum_{q, p=1}^{\mathbf{P}} \stackrel{\circ}{I}_{\varepsilon, q}^{2}(j) \stackrel{\circ}{I}_{\varepsilon, p}(j)-2\right\}\right)^{2}=O\left(\frac{n^{2}}{\mathbf{P}}\right) .
$$


So, $(\tilde{n} \mathbf{P})^{1 / 2} \mathcal{T}_{n}=(\tilde{n} \mathbf{P})^{-1 / 2} \sum_{j=1}^{\tilde{n}} \sum_{p=1}^{\mathbf{P}}\left\{\stackrel{\circ}{I}_{\varepsilon, p}^{2}(j)-1-2 \stackrel{\circ}{I}_{\varepsilon, p}(j)\right\}+o_{p}(1)$ and thus the proof is completed if we show that

$$
\frac{1}{(\tilde{n} \mathbf{P})^{1 / 2}} \sum_{j=1}^{\tilde{n}} \sum_{p=1}^{\mathbf{P}}\left\{\stackrel{\circ}{I}_{\varepsilon, p}^{2}(j)-1-2 \stackrel{\circ}{I}_{\varepsilon, p}(j)\right\} \stackrel{d}{\rightarrow} \mathcal{N}\left(0,4+\kappa_{4}\right)
$$

To that end, we need to check the "generalized" Lindeberg's condition as in Theorem 2 of Phillips and Moon (1999). Indeed, a sufficient condition for that theorem to hold true is that

$$
\mathcal{E}\left(\frac{1}{\mathbf{P}^{1 / 2}} \sum_{p=1}^{\mathbf{P}}\left\{\stackrel{\circ}{I}_{\varepsilon, p}^{2}(j)-1-2 \stackrel{\circ}{I}_{\varepsilon, p}(j)\right\}\right)^{4}<\infty
$$

uniformly in $\mathbf{P}$ and $j$. But this is the case by $C 1$ and that $\mathcal{E} \stackrel{\circ}{I} \varepsilon, p_{8,}^{8}(j)<\infty$. This concludes the proof of the Theorem.

\section{PROOF OF THEOREM 2}

We first show that

$$
\widehat{c}_{j}-c_{j}=w_{j}+\frac{1}{\tilde{n}} \sum_{\ell=1}^{\tilde{n}}\left(\frac{1}{\mathbf{P}} \sum_{p=1}^{\mathbf{P}} R_{p}(\ell)\right) \cos \left(j \lambda_{\ell}\right)+O_{p}\left(\mathbf{P}^{-2} n^{-1 / 2}\right), \text { for } j \geq 1,
$$

where $\mathcal{E}\left|w_{j}\right|^{2}=O\left(n^{-1} \mathbf{P}^{-1}\right)$ and $\mathcal{E}\left(w_{j} w_{k}\right)=0$ for $j \neq k$, and the $O_{p}(\cdot)$ is uniformly in $j \geq 1$.

Indeed denoting $c_{j, n}=\tilde{n}^{-1} \sum_{\ell=1}^{\tilde{n}} \log f(\ell) \cos \left(j \lambda_{\ell}\right)$, by definition

$$
\widehat{c}_{j}-c_{j}=\widehat{c}_{j}-c_{j, n}+O\left(\min \left(n^{-1},\left|c_{j}\right|\right)\right),
$$

because twice continuous differentiability of $\log f(\lambda)$ implies that

$$
\begin{aligned}
\frac{1}{n} \sum_{\ell=1}^{n} \log f(\ell) \cos \left(j \lambda_{\ell}\right)-c_{j} & =\frac{1}{n} \sum_{\ell=1}^{n}\left\{\sum_{k=0}^{\infty} c_{k} e^{i k \lambda_{\ell}}\right\} \cos \left(j \lambda_{\ell}\right)-c_{j} \\
& =O\left(\min \left(n^{-1},\left|c_{j}\right|\right)\right) .
\end{aligned}
$$


Recall that (8) indicates that $c_{k}$ is the $k$ th Fourier coefficient of $\log f(\lambda)$.

Next, using the inequality $\sup _{\ell=1, \ldots, \tilde{n}}\left|a_{p}\right| \leq\left(\sum_{\ell} a_{p}^{q}\right)^{1 / q}$ together with (12), we get that

$$
\begin{aligned}
\mathcal{E} \sup _{\ell=1, \ldots, \tilde{n}}|\widehat{f}(\ell)-f(\ell)|^{2} & \leq \sum_{\ell=1}^{\tilde{n}} \mathcal{E}\left|\frac{1}{\mathbf{P}} \sum_{p=1}^{\mathbf{P}} R_{p}(\ell)\right|^{2}+\left(\sum_{\ell=1}^{\tilde{n}} \mathcal{E}\left(\frac{1}{\mathbf{P}} \sum_{p=1}^{\mathbf{P}} \stackrel{\circ}{I}_{\varepsilon, p}(\ell)\right)^{4}\right)^{1 / 2} \\
& =O\left(n^{-1 / 2} \mathbf{P}^{-1}\right) .
\end{aligned}
$$

So, using that $\mathcal{E}|\widehat{f}(\ell)-f(\ell)|^{2}=O_{p}\left(\mathbf{P}^{-1}\right)$ and (19), we obtain that Taylor's expansion of $\log f(\lambda)$ implies that

$$
\begin{aligned}
\widehat{c}_{j}-c_{j, n}= & \frac{1}{\tilde{n}} \sum_{\ell=1}^{\tilde{n}}\left(\frac{\widehat{f}(\ell)}{f(\ell)}-1\right) \cos \left(j \lambda_{\ell}\right) \\
& +\frac{1}{\tilde{n}} \sum_{\ell=1}^{\tilde{n}}\left(\frac{\widehat{f}(\ell)}{f(\ell)}-1\right)^{2} \cos \left(j \lambda_{\ell}\right)+O_{p}\left(\mathbf{P}^{-2} n^{-1 / 2}\right),
\end{aligned}
$$

where the $O_{p}(\cdot)$ is uniformly in $j$. Using (10) and (12), we have that

$$
\begin{aligned}
\widehat{c}_{j}-c_{j, n}= & \frac{1}{\tilde{n}} \sum_{\ell=1}^{\tilde{n}}\left(\frac{1}{\mathbf{P}} \sum_{p=1}^{\mathbf{P}} \stackrel{\circ}{I}_{\varepsilon, p}(\ell)\right) \cos \left(j \lambda_{\ell}\right) \\
& +\frac{1}{\tilde{n}} \sum_{\ell=1}^{\tilde{n}}\left(\frac{1}{\mathbf{P}} \sum_{p=1}^{\mathbf{P}} \stackrel{\circ}{I}_{\varepsilon, p}(\ell)\right)^{2} \cos \left(j \lambda_{\ell}\right) \\
& +\frac{1}{\tilde{n}} \sum_{\ell=1}^{\tilde{n}}\left(\frac{1}{\mathbf{P}} \sum_{p=1}^{\mathbf{P}} R_{p}(\ell)\right) \cos \left(j \lambda_{\ell}\right)+O_{p}\left(\mathbf{P}^{-1} n^{-1}\right),
\end{aligned}
$$

where the $O_{p}(\cdot)$ is uniformly in $j \geq 1$. Because $\sum_{\ell=1}^{\tilde{n}} \cos \left(j \lambda_{\ell}\right)=0$ if $j \geq 1$, we have that proceeding as in the proof of Theorem 1, the second moment of the first and second terms on the right of the last displayed expression is $O\left((n \mathbf{P})^{-1}+\left(n \mathbf{P}^{2}\right)^{-1}\right)$. From here it is standard to conclude (17). Finally, we have that

$$
\mathcal{E}\left(w_{j} w_{k}\right)=0 \text { if } j \neq k,
$$


which follows easily from the fact that the moments of either $\mathbf{P}^{-1} \sum_{p=1}^{\mathbf{P}} \stackrel{\circ}{I}_{\varepsilon, p}(\ell)$ or $\left(\mathbf{P}^{-1} \sum_{p=1}^{\mathbf{P}} \stackrel{\circ}{I}, p_{\varepsilon}(\ell)\right)^{2}$ are independent of $\ell$. Recall that $\left\{\varepsilon_{t, p}\right\}_{t \in \mathbb{Z}}$ are independent sequences of random variables and $\sum_{\ell=1}^{\tilde{n}} \cos \left(j \lambda_{\ell}\right)=0$ if $j \geq 1$. It is worth observing that in particular we have shown that

$$
\widehat{\sigma}_{\varepsilon}^{2}-\sigma_{\varepsilon}^{2}=\left(\widehat{c}_{0}-c_{0}\right)(2 \pi) e^{c_{0}}+o_{p}(1) .
$$

Let $A_{n}(j)=: \exp \left\{\sum_{u=1}^{\tilde{n}} c_{u} e^{-i u \lambda_{j}}\right\}$. Then uniformly in $j$, we have that

$$
\begin{aligned}
\log \left(\widehat{A}(j) / A_{n}(j)\right)= & \sum_{u=1}^{\tilde{n}}\left(\widehat{c}_{u}-c_{u, n}\right) e^{-i u \lambda_{j}} \\
= & \sum_{u=1}^{\tilde{n}}\left\{w_{u}+\frac{1}{\tilde{n}} \sum_{\ell=1}^{\tilde{n}} \cos \left(u \lambda_{\ell}\right) \frac{1}{\mathbf{P}} \sum_{p=1}^{\mathbf{P}} R_{p}(\ell)\right\} e^{-i u \lambda_{j}} \\
& +O_{p}\left(\mathbf{P}^{-2} n^{1 / 2}+\mathbf{P}^{-1}\right) .
\end{aligned}
$$

Next,

$$
\begin{aligned}
& \mathcal{E}\left(\sum_{u=1}^{\tilde{n}} \frac{1}{\tilde{n}} \sum_{\ell=1}^{\tilde{n}}\left(\frac{1}{\mathbf{P}} \sum_{p=1}^{\mathbf{P}} R_{p}(\ell)\right) \cos \left(u \lambda_{\ell}\right) e^{-i u \lambda_{j}}\right)^{2} \\
= & \mathcal{E}\left(\sum_{\ell=1}^{\tilde{n}}\left(\frac{1}{\mathbf{P}} \sum_{p=1}^{\mathbf{P}} R_{p}(\ell)\right) \frac{1}{\tilde{n}} \sum_{u=1}^{\tilde{n}} \cos \left(u \lambda_{\ell}\right) e^{-i u \lambda_{j}}\right)^{2} \\
= & \mathcal{E}\left(\sum_{\ell=1}^{\tilde{n}}\left(\frac{1}{|\ell-j|_{+}}+\frac{1}{|\ell+j|}\right) \frac{1}{\mathbf{P}} \sum_{p=1}^{\mathbf{P}} R_{p}(\ell)\right)^{2}=O\left(\mathbf{P}^{-1} n^{-1}\right),
\end{aligned}
$$

where $|a|_{+}=\max (1,|a|)$, using (12). Finally, proceeding as with the last expression, it is easy to observe that

$$
\mathcal{E}\left(\sum_{u=1}^{\tilde{n}} w_{u} \cos \left(u \lambda_{\ell}\right) e^{-i u \lambda_{j}}\right)^{2}=O\left(\mathbf{P}^{-1}\right)
$$

Next twice continuous differentiability of $f(\lambda)$ implies that

$$
A_{n}(j)-A(j)=A(j)^{-1} \exp \left\{-\sum_{u=n}^{\infty} c_{u} e^{-i u \lambda_{j}}\right\}=O\left(n^{-2}\right) .
$$


So that, Taylor expansion of $A^{-1}(j) \widehat{A}(j)-1$ indicates that

$$
\begin{aligned}
A^{-1}(j) \widehat{A}(j)-1 & =b(j)+O_{p}\left(\mathbf{P}^{-2} n^{1 / 2}+\mathbf{P}^{-1}\right) \\
\mathcal{E}\left(b(j)^{2}\right) & =O\left(\mathbf{P}^{-1}\right)
\end{aligned}
$$

Now, by definition

$$
\begin{aligned}
\widehat{\varepsilon}_{t, p}-\varepsilon_{t, p}= & \frac{1}{n} \sum_{j=1}^{n} e^{i t \lambda_{j}}\left\{\widehat{A}(j) \sum_{s=1}^{n} x_{s, p} e^{-i s \lambda_{j}}-\sum_{s=1}^{n} \varepsilon_{s, p} e^{-i s \lambda_{j}}\right\} \\
= & \frac{1}{n} \sum_{j=1}^{n} e^{i t \lambda_{j}}\left(\widehat{A}(j) A^{-1}(j)-1\right)\left\{A(j) \sum_{s=1}^{n} x_{s, p} e^{-i s \lambda_{j}}-\sum_{s=1}^{n} \varepsilon_{s, p} e^{-i s \lambda_{j}}\right\} \\
& +\frac{1}{n} \sum_{j=1}^{n} e^{i t \lambda_{j}}\left(\widehat{A}(j) A^{-1}(j)-1\right) \sum_{s=1}^{n} \varepsilon_{s, p} e^{-i s \lambda_{j}} \\
& +\frac{1}{n} \sum_{j=1}^{n} e^{i t \lambda_{j}}\left\{A(j) \sum_{s=1}^{n} x_{s, p} e^{-i s \lambda_{j}}-\sum_{s=1}^{n} \varepsilon_{s, p} e^{-i s \lambda_{j}}\right\} .
\end{aligned}
$$

By Barlett's decomposition, see Brockwell and Davis (2000), it is clear that the first term on the right is $O_{p}\left(\mathbf{P}^{-1}\right)$ uniformly in $t \geq 1$. The second term on the right of (20) is

$$
\begin{aligned}
& \frac{1}{n^{1 / 2}} \sum_{j=1}^{n} e^{i t \lambda_{j}}\left(\frac{1}{\tilde{n}} \sum_{\ell=1}^{\tilde{n}} \cos \left(j \lambda_{\ell}\right) \frac{1}{\mathbf{P}} \sum_{p=1}^{\mathbf{P}} \stackrel{\circ}{I}_{\varepsilon, p}(\ell)\right) \frac{1}{n^{1 / 2}} \sum_{s=1}^{n} \varepsilon_{s, p} e^{-i s \lambda_{j}} \\
& +O_{p}\left(n^{1 / 2} / \mathbf{P}\right)
\end{aligned}
$$

uniformly in $t=1, \ldots, n$. It is clear that using the inequality $\sup _{\ell=1, \ldots, \tilde{n}}\left|a_{p}\right| \leq$ $\left(\sum_{\ell} a_{p}^{q}\right)^{1 / q}$, the last displayed expression is $O_{p}\left(\mathbf{P}^{-1 / 2}+n^{1 / 2} / \mathbf{P}\right)$ uniformly in $t \geq 1$. So, it remains to examine the third term on the right of (20), which is

$$
\frac{1}{n^{1 / 2}} \sum_{j=1}^{n} e^{i t \lambda_{j}}\left\{A(j) \frac{1}{n^{1 / 2}}\left\{\sum_{\ell=n+1}^{\infty}+\sum_{\ell=0}^{n}\right\} b_{\ell} e^{-i \ell \lambda_{j}}\left[\sum_{s=1-\ell}^{n-\ell}-\sum_{s=1}^{n}\right] \varepsilon_{s, p} e^{-i s \lambda_{j}}\right\}
$$

using formulae (10.3.12) of Brockwell and Davis (1991). Using the standard result $\mathcal{E}\left(w_{\varepsilon, p}(j) w_{\varepsilon, p}(-k)\right)=\mathcal{I}(j=k)$, we have that

$$
\frac{1}{n^{1 / 2}} \sum_{j=1}^{n-1} e^{i t \lambda_{j}}\left\{A(j) \frac{1}{n^{1 / 2}} \sum_{\ell=n+1}^{\infty} b_{\ell} e^{-i \ell \lambda_{j}}\left[\sum_{s=1-\ell}^{n-\ell}-\sum_{s=1}^{n}\right] \varepsilon_{s, p} e^{-i s \lambda_{j}}\right\}=O_{p}\left(n^{-1}\right)
$$


because by $C 1, b_{\ell}=o\left(\ell^{-2}\right)$. Also, it is clear using the previous arguments that the last displayed expression is $o_{p}\left(n^{-1 / 2}\right)$ uniformly in $t \geq 1$. So, it remains to examine the contribution due to $\sum_{\ell=0}^{n}$, which using the change of subindex $s-n=r$, it is straightforward to notice that it suffices to examine

$$
\begin{aligned}
& \frac{1}{n^{1 / 2}} \sum_{j=1}^{n} e^{i t \lambda_{j}}\left\{A(j) \frac{1}{n^{1 / 2}} \sum_{\ell=0}^{n} b_{\ell} e^{-i \ell \lambda_{j}} \sum_{s=1-\ell}^{n-\ell} \varepsilon_{s, p} e^{-i s \lambda_{j}}\right\} \\
= & \frac{1}{n^{1 / 2}} \sum_{j=1}^{n} e^{i t \lambda_{j}}\left\{\sum_{q=0}^{\infty} a_{q} e^{-i q \lambda_{j}} \frac{1}{n^{1 / 2}} \sum_{\ell=0}^{n} b_{\ell} e^{-i \ell \lambda_{j}} \sum_{s=1-\ell}^{n-\ell} \varepsilon_{s, p} e^{-i s \lambda_{j}}\right\} \\
= & \left\{\sum_{q=0}^{\infty} a_{q} e^{-i q \lambda_{j}} \frac{1}{n^{1 / 2}} \sum_{\ell=0}^{n} b_{\ell} e^{-i \ell \lambda_{j}} \sum_{s=1-\ell}^{n-\ell} \varepsilon_{s, p} e^{-i s \lambda_{j}} \frac{1}{n} \sum_{j=1}^{n} e^{-i(q+\ell+s-t) \lambda_{j}}\right\} \\
= & \sum_{\ell=0}^{t} b_{\ell} \sum_{s=1-\ell}^{n-\ell} a_{t-(\ell+s)} \varepsilon_{s, p}+\sum_{\ell=t}^{n} b_{\ell}\left\{\sum_{s=1-\ell}^{-t}+\sum_{s=-t}^{0}\right\} a_{t-(\ell+s)} \varepsilon_{s, p}
\end{aligned}
$$

because $\sum_{j=1}^{n} e^{-i(q+\ell+s-t) \lambda_{j}}=n \mathcal{I}(q+\ell+s=t)$. The second and third terms are $o_{p}\left(t^{-1}\right)$ by summability of $a_{q}$ and $C 1$. Finally, the first absolute moment of the first term is easily seen to be $o\left(t^{-1}\right)$ uniformly in $t=1, \ldots, n$.

So, we conclude that uniformly in $t=1, \ldots, n, \widehat{\varepsilon}_{t, p}-\varepsilon_{t, p}=o_{p}\left(t^{-1}\right)$. From here it is standard to conclude the proof of the theorem.

\section{CONCLUSION}

In this paper we have described and examined a simple test for equality of an increasing number of spectral density functions of unspecified functional form. One interesting aspect of the test is that, even without knowledge of the spectral density function under the null, there were no need to choose any bandwidth or smoothing parameter for its implementation. This is possible by using all the information given in data, and in particular that the number of sequences $\mathbf{P}$ also increases without limit. The implementation of the test is straightforward after we make use of the fast Fourier transform. A second interesting aspect of the test is that its asymptotic 
distribution is a normal random variable, although its asymptotic variance depends on the "average" fourth cumulants of the sequences. So the implementation of the test might be thought to be challenging as to provide an estimator of this average of the fourth cumulant of the innovation sequences $\left\{\varepsilon_{t, p}\right\}_{t \in \mathbb{Z}, p \in \mathbb{N}}$. This is the case as we have not made any specification on the dynamic structure of the sequences $\left\{x_{t, p}\right\}_{t \in \mathbb{Z}, p \in \mathbb{N}}$. However, after realizing that all we need is to provide a consistent estimator of the average fourth cumulant instead of the fourth cumulants for each of the individual sequences, we suggest a very simple estimator based on the canonical decomposition of the spectral density function as given in Whittle (1954), see also Hannan (1970) or Brillinger (1981) for more details.

There are several interesting issues worth examining as those already mentioned in the introduction. One of them is how we can extend this methodology to the situation where the sample sizes for the different sequences are not necessarily the same. We believe that the methodology in the paper can be implemented after some smoothing has been put in placed, for instance, via splines. A second relevant extension is what happens when there exists dependence among the sequences. This scenario might be the rule rather than the exception with, say, spatio-temporal data or with large panel data sets with cross-section dependence across individuals. That is, if we denote $\gamma_{p, q}(t)$ the dependence between $\left\{\varepsilon_{t, p}\right\}_{t \in \mathbb{Z}}$ and $\left\{\varepsilon_{t, q}\right\}_{t \in \mathbb{Z}}, p, q \in \mathbb{N}$, the question is how are our results going to change in this scenario? We conjecture that, after inspection of our proofs, a condition such as $\mathbf{P}^{-1} \sum_{p, q=1}^{\mathbf{P}}\left|\gamma_{p, q}(t)\right| \leq C<\infty$ uniformly in $t$ will suffice for the main conclusions in Theorem 1 to go through. However, the technical details to accomplish this and in particular those to obtain a consistent estimator of the asymptotic variance might be cumbersome and lengthy. We envisage, though, that this is possible via bootstrap methods using results given in Section 2 together with those obtained by Chang and Ogden (2009) to be able to obtain a simple computational estimator of the long run variance of the test. However the 
details are beyond the scope of this paper. Finally, there are a couple of problems, mentioned in the introduction, where the methods developed in the paper can be used. One of them was on testing for stationarity of the covariance structure of a sequence of random variables, being the second one on testing for separability of the covariance function in a spatio-temporal data set.

\section{REFERENCES}

[1] Brillinger, D.R. (1981). Time Series, Data Analysis and Theory. Holden-Day, San Francisco.

[2] Brockwell, P.J. And Davis, R.A. (1991). Time Series: Theory and Methods. Springer-Verlag, New York.

[3] Chang, C. and Ogden, R.T. (2009). Bootstrapping sums of independent but not identically distributed continuous processes with applications to functional data. Journal of Multivariate Analysis, 100, 1291-1303.

[4] Contes, D.S. And Diggle, P.J. (1986). Test for computing two estimated spectral densities. Journal of Time Series Analysis, 7, 7-20.

[5] Degras, D. Xu, Z. Zhang, T. And Wu, W.B. (2012). Testing parallelism between trends in multiple time series. IEEE Transactions on Signal Processing, 60, 10871097.

[6] Detter, H. And Paparoditis, E. (2009). Bootstrapping frequency domain tests in multivariate time series with an application to testing equality of spectral densities. Journal of Royal Statistical Society, Ser. B, 71, 831-857.

[7] Diggle, P.J. And Fisher, N.I. (1991). Nonparametric comparison of cumulative 
periodograms. Applied Statistics, 40, 423-434.

[8] Ferraty, F. And Vieu, P. (2006). Nonparametric functional data analysis: Theory and practice. Springer-Verlag. Berlin.

[9] Fuentes, M. (2006). Testing for separability of spatial-temporal covariance functions. Journal of Statistical Planning and Inference, 136, 447-466.

[10] Grenander, U. and Rosenblatt, M. (1957). Statistical Analysis of Stationary Time Series. John Wiley, New York.

[11] Hannan, E.J. (1970). Multiple Time Series. Wiley and Sons, New York.

[12] Härdle, W. and Marron, T.S. (1990). Semiparametric comparison of regression curves. Annals of Statistics, 18, 63-89.

[13] Hidalgo, J. and Yajima, Y. (2002). Prediction and signal extraction of strongly dependent processes in the frequency domain. Econometric Theory, 18, 584-624.

[14] Phillips, P.C.B. and Moon, R. (1999). Linear regression limit theory for nonstationary panel data. Econometrica 67, 1057-1111.

[15] Pinkse, J. And Robinson, P.M. (1995). Pooling nonparametric estimates of regression functions with a similar shape. In Statistical Methods in Econometrics and Quantitative Economics: A volume in honour of C.R. Rao, G.S. Maddala, P.C.B. Phillips and T.N. Srinivisan, eds. pp. 172-197.

[16] Whittle, P. (1954): "On stationary processes in the plane," Biometrika, 41, 434449.

[17] Zhang, Y, Su, L. and Phillips,P.C.B. (2012). Testing for common trends in semiparametric panel data models with fixed effects. The Econometrics Journal, 15, 56-100. 
[18] Zhu, J. Lahiri, S.N. and Cressie, N. (2002). Asymptotic inference for spatial CDFS over time. Statistica Sinica, 12, 843-861. 